\title{
Are clinical pharmacology studies still needed in childhood acute lymphoblastic leukemia?
}

\section{Valentino Conter ${ }^{1}$ and Francesco Ceppi ${ }^{2}$}

\author{
${ }^{1}$ Pediatric Hemato-Oncology, Fondazione MBBM, University Milano Bicocca, Ospedale San Gerardo, Monza, Italy and ${ }^{2}$ Pediatric \\ Hematology-Oncology Unit, Division of Pediatrics, Department Woman-Mother-Child, University Hospital of Lausanne \& University \\ of Lausanne, Lausanne, Switzerland
}

E-mail:VALENTINO CONTER - valentino.conter@gmail.com

doi:10.3324/haematol.2021.279059

I $\mathrm{n}$ this issue of Haematologica, Karol et al. report a study on dose intensities for all drugs in two consecutive acute lymphoblastic leukemia (ALL) clinical trials at St. Jude Children's Research Hospital, which differed in their asparaginase formulation and intensity. ${ }^{1}$ The amount of data is impressive, with more than 500,000 dosing records. The main message of the manuscript is that the lack of benefit from increased asparaginase intensity may be due to the decrease of dose intensity of other drugs, induced by the additional treatment with asparaginase.

It is widely recognized that intensity of chemotherapy delivered has an impact on outcome and that drug interactions, which are difficult to assess, can influence anticancer activity and acute and/or late toxicity too. The fast improvement of outcome in childhood ALL in the last three decades of the last century were strictly associated with progressive treatment intensity. Dr. Riehm was the pioneer in this historical process, which was thereafter pursued by all major pediatric oncology groups. In the early 1990s, Sallan summarized the Dana-Faber Cancer Institute (DFCI) experience, largely based on treatment intensification with asparaginase, with the words "More is better!", ${ }^{2}$ and Niemeyer (with Riehm and Sallan) suggested that merging the intensive elements of BerlinFrankfurt-Münster (BFM) and DFCI protocols would be a logical program to improve outcomes. ${ }^{3}$ Various attempts were made in this frame, sometimes successfully, such as in the Children's Cancer Study Group (CCSG) study with Augmented BFM. ${ }^{4}$ Most studies did not, however, show any benefit in intensive BFM-oriented protocols, either from additional asparaginase treatment as done in Associazione Italiana Ematologia Oncologia Pediatrica (AIEOP) ALL 9102, European Organization for Research and Treatment of Cancer - Children Leukemia Group (EORTC-CLG) 58951, ${ }^{6}$ Nordic Society of Pediatric Hematology and Oncology (NOPHO) ALL-2008, ${ }^{7}$ and BFM ALL 90 trials, $^{8}$ or from the marked intensification in the Children's Oncology Group (COG) AALL1131 trial with clofarabine, which was interrupted early due to an excess of toxicity. ${ }^{9}$

This general experience has led to a consensus that treatment intensity in childhood ALL may have reached the maximum tolerated doses, so that further improvement can only be obtained by precision medicine based on targeted therapies. However, most children with ALL are cured with conventional chemotherapy, which can be further optimized and tailored thanks to the progressive improvement of biology-based stratification.

The study by Karol et al. shows that room remains for improvement of chemotherapy, although this cannot be achieved by a simple protocol therapy intensification. ${ }^{1}$ Asparaginase is a drug with a unique mechanism of action, and there are no suggested alternatives to replace it in patients who cannot be treated with the drug. DFCI studies showed that these patients have a poorer outcome. In this context it quite interesting the finding that patients with low asparaginase dose intensity, a higher systemic methotrexate dose intensity compensated for the low asparaginase dose intensity. The often neglected and yet very relevant aspect of oral medications administered at home is also of note. In the study reported in this issue, there is the apparent paradox of higher relapse rate associated with higher dose intensity for mercaptopurine, which the authors suggest might reflect low treatment adherence for oral medications at home (not measured in this study), in keeping with the findings of the COG AALL03N1 study, in which it was shown that an adherence rate below $90 \%$ to maintenance therapy was associated with an increased relapse risk. ${ }^{10}$

Although the expectation for further improvements in the treatment of childhood ALL is mostly based on innovative immunological or targeted therapies, pharmacological studies remain crucial to improve the therapeutic index of combinations of antineoplastic agents. To this purpose, it must be considered that simple measurement of duration of treatment phases, incidence of severe adverse effects, and dose intensities of single agents may be inadequate or even misleading. What is needed in order to optimize precision personalized treatment in childhood ALL are comprehensive investigations of compliance/adherence for all drugs, drug interactions and bioavailability, and germline and tumor sensitivity.

\section{Disclosures}

No conflicts of interest to disclose.

\section{Contributions}

The two authors contributed equally.

\section{References}

1. Karol SE, Pei D, Smith CA, et al. Comprehensive analysis of dose intensity of acute lymphoblastic leukemia chemotherapy. Haematologica. 2021;107(2):371-380.

2. Sallan SE, Gelber RD, Kimball V, Donnelly M, Cohen HJ. More is better! Update of Dana-Farber Cancer Institute/Children's Hospital childhood acute lymphoblastic leukemia trials. Haematol Blood Transfus. 1990;33:459-466.

3. Niemeyer CM, Reiter A, Riehm H, Donnelly M, Gelber RD, Sallan SE. Comparative results of two intensive treatment programs for childhood acute lymphoblastic leukemia: the Berlin-FrankfurtMünster and Dana-Farber Cancer Institute protocols. Ann Oncol. 1991;2(10):745-749.

4. Nachman J, Sather HN, Gaynon PS, Lukens JN, Wolff L, Trigg ME. Augmented Berlin-Frankfurt-Munster therapy abrogates the adverse prognostic significance of slow early response to induction chemotherapy for children and adolescents with acute lymphoblastic leukemia and unfavorable presenting features: a report from the Children's Cancer Group. J Clin Oncol. 1997;15(6):2222-2230.

5. Rizzari C, Valsecchi MG, Aricò M, et al. Effect of protracted high- 
dose L-asparaginase given as a second exposure in a Berlin-FrankfurtMünster-based treatment: results of the randomized 9102 intermediate-risk childhood acute lymphoblastic leukemia study--a report from the Associazione Italiana Ematologia Oncologia Pediatrica. J Clin Oncol. 2001;19(5):1297-1303.

6. Mondelaers V, Suciu S, De Moerloose B, et al. Prolonged versus standard native E. coli asparaginase therapy in childhood acute lymphoblastic leukemia and non-Hodgkin lymphoma: final results of the EORTC-CLG randomized phase III trial 58951. Haematologica. 2017;102(10):1727-1738

7. Toft N, Birgens H, Abrahamsson J, et al. Results of NOPHO ALL2008 treatment for patients aged 1-45 years with acute lymphoblastic leukemia. Leukemia. 2018;32(3):606-615.
8. Schrappe M, Reiter A, Ludwig WD, et al. Improved outcome in childhood acute lymphoblastic leukemia despite reduced use of anthracyclines and cranial radiotherapy: results of trial ALL-BFM 90 German-Austrian-Swiss ALL-BFM Study Group. Blood. 2000;95(11):3310-3322

9. Salzer WL, Burke MJ, Devidas M, et al. Toxicity associated with intensive postinduction therapy incorporating clofarabine in the very high-risk stratum of patients with newly diagnosed high-risk Blymphoblastic leukemia: a report from the Children's Oncology Group study AALL1131. Cancer. 2018;124(6):1150-1159.

10. Bhatia S, Landier W, Hageman L, et al. 6MP adherence in a multiracial cohort of children with acute lymphoblastic leukemia: Children's Oncology Group study. Blood. 2014;124(15):2345-2353. 